\title{
The Year 2050
}

\subsection{The Economy of the Jordan Valley in 2050}

Under the scenario and strategy described in this Regional Master Plan, by 2050 the Jordan Valley will be a co-operative, confident and peaceful region with a healthy economy and strong development perspectives for the people living here. They will experience a clean and healthy environment and sufficient flows in the Jordan River to sustain healthy eco-systems. At the same time the river will act as natural water conveyor and source for water supply in the Jordan Valley. Water will be equitably shared among the three riparian countries and the valley will be freely accessible for all nationalities within an appropriate security framework. Local, private and foreign investments will be encouraged due to the stability in the region. In short, there will be an investment climate resulting from the reforms in general, and a conductive regulatory business environment that promotes sustainable development (Figs. 5.1, 5.2 and 5.3).

In 2050 the valley will house around 750,000 people in Jordan, 500,000 people in Palestine and 90,000 people in Israel, who will enjoy their living environment in terms of living, working and recreational conditions. They will live in a comfortable and sustainable urban setting with an average of about 3.5 people per household. There will be about 370,000 household units in the valley, compared to about 65,000 today. This will be the result of substantial investments in urban and infrastructure projects in the range of 3 Billion US Dollar until 2050, with relative smaller housing units than existing today. Meanwhile the roads and infrastructure have been upgrade with adequate traffic safety measures, including efficient public transport, bypasses around urban centers, pedestrian and bicycle sideway capacities and more.

Due to investments in tourism, sustainable agriculture and agri-business, as well as in housing, infrastructure, higher education and public services the people in the valley will enjoy attractive job opportunities. The economy will become more service and high added value oriented, with a higher percentage of people being employed in the service sector. The average income will have risen substantially to about 14,000 USD per person or about 50,000 USD per household in Jordan and Palestine and to about 72,000 USD per person in Israel.

In 2050 the Palestinian economy will experience substantial growth, unrestricted by land use or access and sufficient water resources to meet their demands as described before. This will have synergetic positive impacts on the valley economy at large, due to growth of export and import of goods and knowledge both from Israel and Jordan.

The valley economy in 2050 will strongly benefit from the expanding construction and real estate sector, responsible for the realization of the additionally required infrastructure and urban housing units. This in turn will have an economic spin off to related sectors, such as the stone and marble industries, public utilities, commercial sectors, telecommunication and more. In addition it is expected that land prices rises will contribute substantially to the overall economic growth. This will particularly be the case for the Palestinian land prices in (previous) area C. For instance the World Bank's economic analysis of area $\mathrm{C}$ and its future economy (Orhan Niksic et al. 2014) indicates that current cost per dunum in area $\mathrm{C}$ is around USD 2,000-5,000 compared to USD 150,000 per dunum in area A. These differences will gradually disappear in a future independent Palestine, leading to an increase of average land prices.

The tourism industry will be one of the cornerstones of the valley's economy in 2050 and will largely benefit for the full co-operation among the three riparian partners that is foreseen by then. Up to 5-10 Million of national, regional and international tourists per year will visit the cities, nature parks, the cultural and religious sites and a wide variety of museums established in the valley, leading to an economic growth in the range of 5-10 Billion USD per year. In additional, the expanded urban centers will provide wide variety commercial services. This leads to an estimated 6,000 people in Israel being employed in the tourism and commerce sectors, 40,000 people in Jordan and 33,000 people in Palestine.

By 2050, security related issues in the valley will be managed by a Security Coordination Body, representing key 


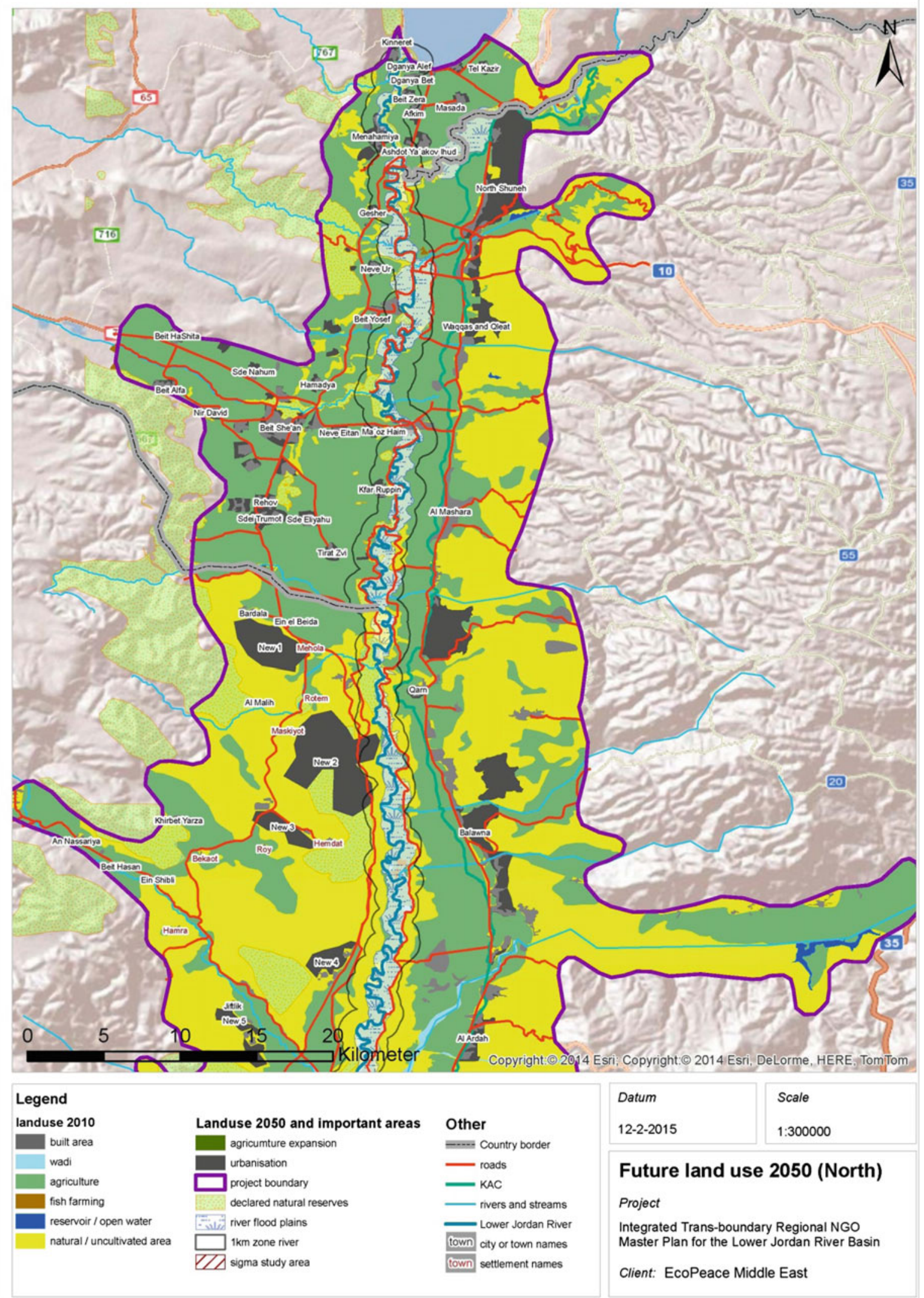

Fig. 5.1 Land use map 2050 for the northern part of the Jordan valley 

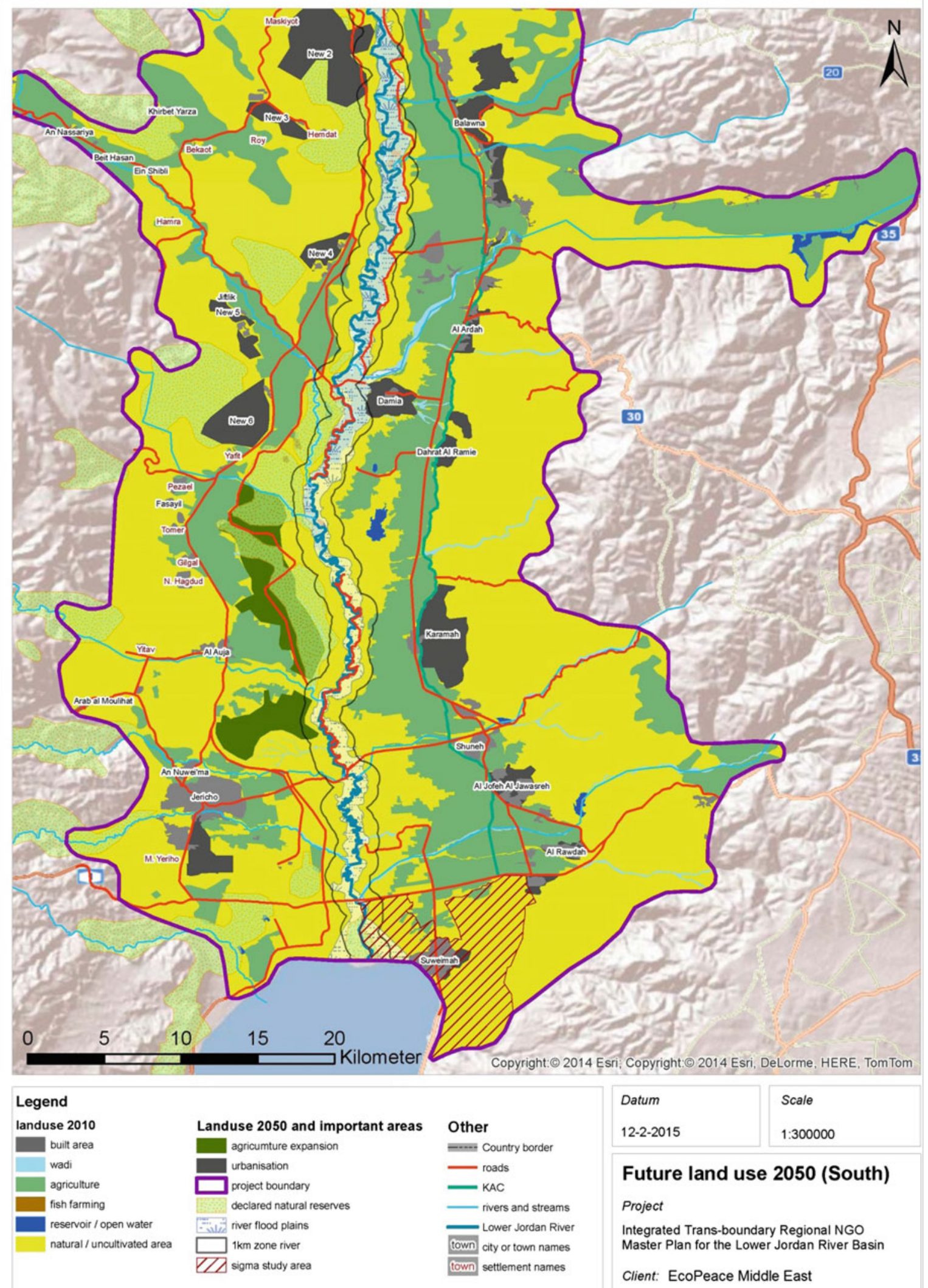

Fig. 5.2 Land use map 2050 for the southern part of the Jordan valley 
Fig. 5.3 Lower part of the Jordan river

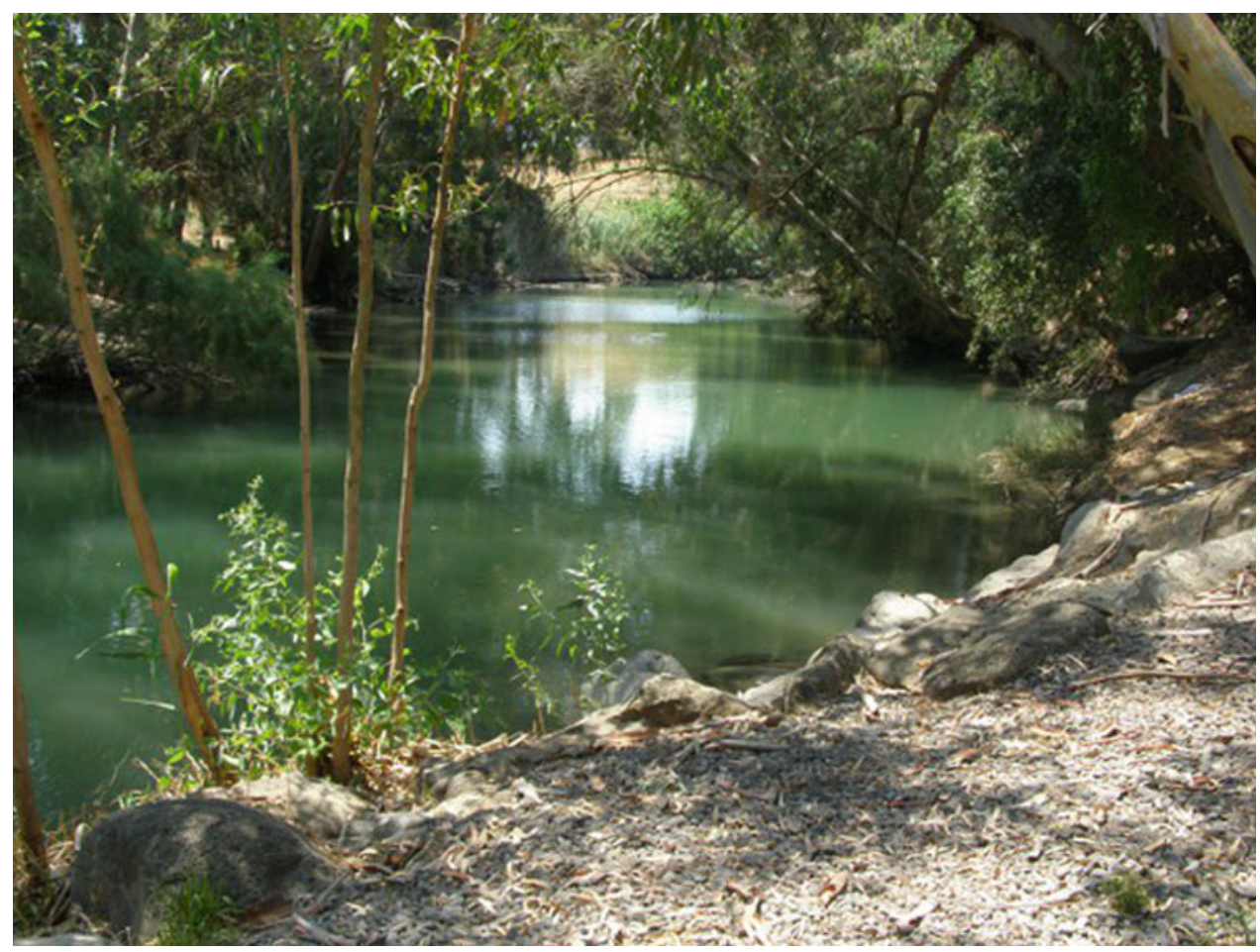

security officials of Israel, Palestine and Jordan. This body will assess and manage the security issues in the valley objectively and professionally, doing justice to the legitimate mobility rights of all people living in the valley, and the legitimate security rights of the people of Israel, Palestine and Jordan.

By 2050 agriculture in the valley has developed into a sustainable and agri-business oriented sector, making efficient use of the valuable water resources and generating high economic revenues as result of efficient extension services, high quality agricultural products and good access to regional and international markets. Due to efficiency measures, about $5 \%$ of the working force will be directly employed in agriculture.

The 2050 fiscal benefits for the riparian partners related to the projected economic growth, will be substantial, including VAT, income and corporate profits related taxes. This will lead to about 10 Billion of tax revenues within the valley. As described earlier in Sect. 4.3, part of these public revenues will be re-invested in public domain infrastructure, public utilities and buildings, education, health care and more. As a result of this, the required annual investment packages identified in this Master Plan will be provided fully by governmental budgets, replacing fully the international donor funds sometime between 2025 and 2030, following later by substantial private sector investments as well.

Finally, in 2050 the three riparian countries will have established an efficient transboundary River Basin Organization, which ensures coordinated water resources management between Jordan, Israel and Palestine on a shared Jordan River
Basin, addressing the legitimate social and economic needs of each of the riparian states, to enable joint development and management of water resources infrastructure among the riparians. It will act as a coordinating water management body for the riparian countries of the LJR, fostering economic co-operation over water resources through a coordinated, transparent and democratic process. Projections of the main economic parameters for 2050 in the valley are presented in Table 5.1. The total combined valley economy will reach 73 Billion USD by 2050 , compared to only around 5 Billion USD in 2010.

The presented economic projections for 2050 depend of many factors beyond the aspects of peace, co-operation and effective basin water management described in this Master Plan. This may include factors like export opportunities, wider global economic and geo-political developments, future trade agreements, level of future education, availability of credits and more. However, assessing these developments would require elaborated regional and global geo-economic projections, which go beyond the scope of this Jordan Valley NGO Master Plan.

\subsection{Land Use in $\mathbf{2 0 5 0}$}

The 2050 Land use plans have been developed on the basis of existing governmental land use allocation plans in Jordan and Israel, including the Israeli governmental northern district outline plan; and the Jordanian Jordan Valley Land Use Plan developed by JVA with support from the US Agency 
for International Development. Next, these plans have been modified on the basis of the 2050 population projections and related land additional land requirements. In Palestine no land use plans have yet been developed, and future plans have been developed after careful evaluation of current land use and various discussions with key stakeholders, including the Governorate and Municipality of Jericho.

Within this study, the following land types have been distinguished:

\section{Uncultivated Lands and Nature Reserves}

These lands have not been developed, and part is controlled and protected as nature reserves. In addition, these lands are important for reasons of groundwater infiltration, as pastures, for sustaining nature and wild life, for recreation purposes and natural landscapes values, and as archaeological potential areas.

For 2050 it is assumed that the nature reserves remain to be protected, in addition an extra $1 \mathrm{~km}$ zone on both sides of the Jordan River. This zone will serve as new aquatic eco-zone and flood plain connected to the rehabilitated Jordan River. In addition, part of the non-protected uncultivated area will be developed in 2050 for urban expansion and infrastructure purposes as indicated in Table 4.7. Furthermore, an additional $43 \mathrm{~km}^{3}$ of uncultivated land in Palestine will be developed for agricultural purposes by 2050 .

\section{Agriculture}

The lands currently used for agriculture have since long been used for reasons of soil fertility and water availability. However, some of the palm plantations have been developed only recently and irrigated with slightly brackish water. In this master plan it is assumed that this agricultural land will also in 2050 remain available for this purpose, and that no additional land in Israel and Jordan has been developed for agriculture. The agricultural lands however will apply more efficient water usage and generate higher economic outputs per $\mathrm{km}^{2}$ in 2050 .

In Palestine, an additional $42.7 \mathrm{~km}^{2}$ of uncultivated land will be added to the agricultural area, and will be supplied with water from the Jordan River in 2050 mainly for palm plantations. The agricultural areas will next be protected in accordance with the following categorization: cat 1: highest value agricultural land with a slope of less than $5 \%$; cat 2 valued agricultural land with a slope between 5 and $20 \%$; cat 3 valued agricultural land with a slope of more than $20 \%$.

\section{Built up Area}

The built up area is defined here as space required for infrastructure and urban areas. The requirements for built up land will grow substantially until 2050 to facilitate a population growth close to 750,000 people in Jordan and 500,000 people in Palestine. As mentioned earlier, it is assumed that the Israeli population will grow from 49,000 today to about 88,000 is the year 2050. See also Table 2.19.
The proposed allocation of built-up area proposed in the 2050 land use plans are fully based on earlier developed land use plans by the responsible Jordanian and Israeli authorities. In Jordan, for instance, substantial urban expansion is foreseen in North Shuneh, near Waqqas and in the middle and southern regions near Qarn, Balawna and Karamah. In addition a new economic development zone, called the Sigma Study Area has been planned in near the Dead Sea. The total built-up area in Jordan will grow from $44.6 \mathrm{~km}^{2}$ today to about $107 \mathrm{~km}^{2}$ in 2050 . In Israel the built up area will grow from about $20 \mathrm{~km}^{2}$ to about $35 \mathrm{~km}^{2}$ in 2050 . These plans include expansion of secondary and primary roads, and linkage to national highway system and public transport requirements.

In Palestine, no regional land use plans were developed that facilitate a population growth up to 500,000 by 2050 . However, the "Jericho Master Plan-A Model for Sustainable Development", was developed in 2014 with support from the Italian Government. This urban master plan aims at preservation the unique historic and cultural tangible and intangible heritage of Jericho; preserve the cultural landscape of the oasis and of the natural landscape of the desert; enhance the role of Palestinian Gate towards Jordan and the rest of the world; reinforce sustainable development and develop sustainable tourism in Jericho.

After careful evaluation of these existing plans, current land use and various discussions with key stakeholders, including the Governorate and Municipality of Jericho, it has been decided to locate the new urban areas in the Northern part of the West Bank. The cities are called "New 1; New 2 and New 3". Furthermore, three areas in the Middle section have been allocated for urban expansion, called "New 4, New 5 and New 6". Finally, the area south of Jericho has been allocated for urban growth. This area is already subject to land development projects, such as the Jericho Gate project and the agro-industrial park being developed here. Totally, the Palestinian built up area will grow from about $25.3 \mathrm{~km}^{2}$ today (including settlements) to $78.8 \mathrm{~km}^{2}$ in 2050 (no more settlements).

\section{Fish Farms}

The foreseen land use by 2050 in Israeli part of the Jordan Valley is based on Israel's governmental northern district outline plan. This plan does not include extensive fish ponds anymore, since it is assumed that some turned into intensive closed system sustainable ponds with elimination of all pollution related problems, and some will have developed alternative agricultural activities instead.

\section{Water Reservoirs, Wadis}

In the proposed 2050 land use maps the lands allocated today for water reservoirs and wadis will remain to be used for these purposes in 2050. 


\subsection{Water Related Impacts in $\mathbf{2 0 5 0}$}

This section provides a description of the water model that has been developed for the Jordan Valley under this study. The model has been constructed using the "Water Evaluation and Planning" (WEAP) software. This model WEAP has been built upon earlier models that have been developed for the area, including the WEAP model for the Roadmap for the Rehabilitation of the Lower part of the Jordan River, the Harmonized Water Database for the Jordan Valley, the Model for Water Supply and Demand for Effective Water Management Allocation in the Jordan Valley, and the WEAP Model for an Integrated Approach to Sustainable Management of the Jordanian Water Resources under Global Change by Glowa.

WEAP is based on the principle of closing the water balance in a valley. It provides a model to understand the balance between the total water resources, and the total water consumption, leading to a model for the monthly and annual flows in the Lower part of the Jordan River, as well as the salinity levels in the river. A preliminary step to develop future planning alternatives is creating a model of the present state, or current account.

General issues relate to the river modeling, including the timeframe, salinity, direct contact between groundwater and the LJR, runoff, and evapotranspiration from the LJR.

\section{Time Frame}

The hydrological year taken in evaluation is the year 2050, from October 1st 2049 until September 30th 2050 of the next year, with monthly model steps in between. The hydrological data include the projections of available water resources against protected climate impacts, including reduction in rainfall and increased evaporation rates.

\section{Projected Water Demands}

The projected water demands that have been considered within valley are presented in the next table. Details of these demands have been presented before.

As in Table 5.1, the proposed water resources will be more of less sufficient to provide the required water demands throughout the Jordan Valley by the year 2050.

\subsection{The Jordan River in $\mathbf{2 0 5 0}$}

\subsubsection{Assumptions}

In the vision for 2050 the Jordan River will play a crucial and multi-functional role. This implies that the water in the river will serve different important functions at the same time, in terms of sustaining ecology, supporting tourism and related economic development, and conveying and supplying water throughout the valley, particularly for Jordan and
Palestine. However, the Jordan River is a dynamic natural water body, in which water flow and quality depend on a complex and time dependent interaction between inflow, abstractions, evaporation and groundwater flows.

To sustain the ecological conditions in the river, WEDO/EcoPeace would ideally like to see a non-polluted flow in the Jordan River with a minimum of $400 \mathrm{MCM} / \mathrm{year}$, including the outflow into the Dead Sea (Tables 5.1, 5.2, 5.3 and 5.4).

In order to meet the water demands presented above, and to reach the flow targets in 2050 , the following assumptions have been made:

- By 2050 some $238 \mathrm{MCM} /$ year of water will be released into the Jordan River from the Sea of Galilee. This implies that Israel will have reduced pumping water from the Sea of Galilee into the National Water Carrier, and will have replaced this by other resources, such as desalinated seawater. However, it is assumed that Israel will maintain supplying its domestic and agricultural water needs in the valley from local water resources. The monthly sequence of this release shall be managed in line with the river annual flow predictions (see Table 5.5) and the annual water demands (Tables 5.6, 5.7 and 5.8).

- Meanwhile the Sea of Galilee will be kept on a medium water level between the top and bottom red lines (What is called now the "green line" by the IWA)

Table 5.1 Economic parameters Jordan valley by 2050

\begin{tabular}{|c|c|c|c|}
\hline 2050 & Jordan & Israel & Palestine \\
\hline Average household size (persons) & 3.5 & 3.3 & 3.5 \\
\hline Average income pppy, USD & 14,000 & 72,339 & 14,000 \\
\hline Employment agriculture (\%) & $5.00 \%$ & $1.60 \%$ & $5.00 \%$ \\
\hline Employment manufacturing/constr & $17.00 \%$ & $16.50 \%$ & $17.00 \%$ \\
\hline Employment tourism/commerce & $20.00 \%$ & $19.50 \%$ & $20.00 \%$ \\
\hline Employment transportation & $3.80 \%$ & $3.80 \%$ & $3.80 \%$ \\
\hline Employment services/others & $54.20 \%$ & $58.60 \%$ & $54.20 \%$ \\
\hline GDP per person, USD & 42,000 & 100,000 & 42,000 \\
\hline Total national inhabitants & 593,189 & 88,200 & 502,531 \\
\hline Total foreign population & 150,000 & 0 & 0 \\
\hline Number of households & 199,483 & 26,727 & 143,580 \\
\hline Built up area requirements $\left(\mathrm{km}^{2}\right)$ & 107.0 & 35.7 & 78.8 \\
\hline Total income (billion USD/year) & 9.35 & 6.38 & 7.04 \\
\hline Employees agriculture & 9,886 & 470 & 8,376 \\
\hline Employees manufacturing/construction & 33,614 & 4,851 & 28,477 \\
\hline Employees tourism/commerce & 39,546 & 5,733 & 33,502 \\
\hline Employees transportation & 7,514 & 1,117 & 6,365 \\
\hline Employees services/others & 107,170 & 17,228 & 90,791 \\
\hline GDP Jordan valley (billion/year) & 29.9 & 20.4 & 22.5 \\
\hline
\end{tabular}


Table 5.2 Projected land use $\left(\mathrm{km}^{2}\right)$

\begin{tabular}{|c|c|c|c|c|}
\hline Area $\left(\mathrm{km}^{2}\right)$ & Israel & Jordan & Palestine & Total \\
\hline \multicolumn{5}{|l|}{2010} \\
\hline $\begin{array}{l}\text { Uncultivated/nature } \\
\text { reserves }\end{array}$ & 61.9 & 810.3 & 671.3 & $1,543.5$ \\
\hline Agriculture & 178.3 & 451.8 & 173.0 & 803.1 \\
\hline Built up area & 19.6 & 44.6 & 25.3 & 89.6 \\
\hline Fish farms & 21.5 & 0.7 & 0.3 & 22.6 \\
\hline Water reservoirs & 0.61 & 5.55 & 0.26 & 6.43 \\
\hline Wadis & 5.3 & 24.2 & 13.8 & 43.24 \\
\hline Total & 287.2 & $1,337.2$ & 884.0 & $2,508.4$ \\
\hline \multicolumn{5}{|l|}{2050} \\
\hline $\begin{array}{l}\text { Uncultivated/nature } \\
\text { reserves }\end{array}$ & 45.8 & 747.9 & 575.1 & $1,368.8$ \\
\hline Agriculture & 199.8 & 451.8 & 215.7 & 845.9 \\
\hline Built up area & 35.7 & 107.0 & 78.8 & 221.5 \\
\hline Fish farms & 0 & 0.7 & 0.3 & 22.6 \\
\hline Water reservoirs & 0.61 & 5.55 & 0.26 & 6.43 \\
\hline Wadis & 5.3 & 24.2 & 13.8 & 43.24 \\
\hline Total & 287.2 & $1,337.2$ & 884.0 & $2,508.4$ \\
\hline
\end{tabular}

Table 5.3 Total water demands in the Jordan valley

\begin{tabular}{l|l|l|l}
\hline $\begin{array}{l}\text { Total domestic water } \\
\text { demands LJRB }(C M)\end{array}$ & 2010 & 2025 & 2050 \\
\hline Jordan domestic & $22,239.720$ & $29,843.979$ & $51,955.104$ \\
\hline $\begin{array}{l}\text { From LJR basin to } \\
\text { Amman, north gov. }\end{array}$ & $60,000.000$ & $80,000.000$ & $100,000.000$ \\
\hline Israel domestic & $4,410.000$ & $5,197.920$ & $7,056.000$ \\
\hline Palestine domestic & $3,922.890$ & $9,903.915$ & $40,202.480$ \\
\hline Total & $90,572.610$ & $124,945.814$ & $199,213.584$ \\
\hline $\begin{array}{l}\text { Total agricultural water } \\
\text { demands LJRB }(\text { CM })\end{array}$ & 2010 & 2025 & 2050 \\
\hline Jordan agriculture & $276,258.306$ & $276,258.306$ & $276,258.306$ \\
\hline Israel agriculture & $151,652.000$ & $151,652.000$ & $151,652.000$ \\
\hline Palestine agriculture & $85,170.469$ & $125,170.470$ & $125,170.470$ \\
\hline Total & $513,080,775$ & $553,080.776$ & $553,080.776$ \\
\hline $\begin{array}{l}\text { Total water demands LJR } \\
\text { basin }\end{array}$ & $603,653.385$ & $678,026.589$ & $752,294.360$ \\
\hline $\begin{array}{l}\text { Total water resources } \\
\text { (MCM/year) }\end{array}$ & 506.18 & 658.67 & 744.34 \\
\hline Total deficit (MCM/year) & 97.47 & 19.35 & 7.95 \\
\hline & & & \\
\hline
\end{tabular}

- All pollution flowing into the Jordan River will have ceased by 2025 . This implies fully treatment of all wastewater, full sanitary solid waste management in the Jordan, Israel and Palestine parts of the valley, and diverting salt water flows around the main part of the river. However, termination of all wastewater and waste
Table 5.4 Jordanian water demands and supply in the Jordan valley

\begin{tabular}{|c|c|c|c|c|}
\hline \multicolumn{2}{|c|}{ Total domestic water demands } & \multirow{2}{*}{$\begin{array}{l}2010 \\
6,536.580\end{array}$} & \multirow{2}{*}{$\begin{array}{l}2025 \\
10,509.846\end{array}$} & \multirow{2}{*}{$\begin{array}{l}2050 \\
20,917.056\end{array}$} \\
\hline JMD 1 & Agwhar Shamaliyah & & & \\
\hline JMD 2 & Deir al Alla/Balqa & $4,075.500$ & $6,552.796$ & $13,041.600$ \\
\hline JMD 3 & Shoonah/Janoobiyah & $4,217.640$ & $6,781.336$ & $13,496.448$ \\
\hline JMD 4 & Foreign population & $7,410.000$ & $6,000.000$ & $4,500.000$ \\
\hline JMD 5 & $\begin{array}{l}\text { To Amman (and } \\
\text { northern governorate) }\end{array}$ & $60,000.000$ & $80,000.000$ & $100,000.000$ \\
\hline \multicolumn{2}{|l|}{ Total } & $82,239.720$ & $109,843.979$ & $151,955.104$ \\
\hline \multirow{2}{*}{\multicolumn{2}{|c|}{ Total agricultural water demands }} & \multirow[t]{2}{*}{2010} & \multicolumn{2}{|l|}{ CM/year } \\
\hline & & & 2025 & 2050 \\
\hline JAD1-4 & $\begin{array}{l}\text { Zone } 1(115,300 \\
\text { dunum) }\end{array}$ & $103,596.865$ & $103,596.865$ & $103,596.865$ \\
\hline JAD5-8 & Zone 2 (74,959 dunum) & $107,169.170$ & $107,169.170$ & $107,169.170$ \\
\hline JAD9-16 & $\begin{array}{l}\text { Zone } 3(120,835 \\
\text { dunum) }\end{array}$ & $65,492.271$ & $65,492.271$ & $65,492.271$ \\
\hline \multicolumn{2}{|c|}{ Total } & $276,258.306$ & $276,258.306$ & $276,258.306$ \\
\hline \multicolumn{2}{|c|}{ Total water demands (CM/year) } & $358,498.026$ & $386,102.285$ & $428,213.410$ \\
\hline \multicolumn{5}{|c|}{ This water is will be supplied to: 2050} \\
\hline \multicolumn{2}{|c|}{$\begin{array}{l}\text { Total Domestic Water Supply } \\
(\text { (CM/year) }\end{array}$} & 2010 & 2025 & 2050 \\
\hline JMD 1 & Agwhar Shamaliyah & $6,536.580$ & $10,509.846$ & $20,917.056$ \\
\hline JMD 2 & Deir al Alla/Balqa & $4,075.500$ & $6,552.796$ & $13,041.600$ \\
\hline JMD 3 & Shoonah/Janoobiyah & $4,217.640$ & $6,781.336$ & $13,496.448$ \\
\hline JMD 4 & $\begin{array}{l}\text { Informal population } \\
\text { (according to JVA) }\end{array}$ & $7,410.000$ & $6,000.000$ & $4,500.000$ \\
\hline JMD 5 & $\begin{array}{l}\text { To Amman/Northern } \\
\text { Governorate }\end{array}$ & $60,000.000$ & $80,000.000$ & $100,000.000$ \\
\hline \multicolumn{2}{|c|}{ Domestic Subtotal } & $82,239.720$ & $109,843.979$ & $151,955.104$ \\
\hline \multirow{2}{*}{\multicolumn{2}{|c|}{ Total agricultural water supply }} & \multirow[t]{2}{*}{2010} & \multicolumn{2}{|l|}{ CM/year } \\
\hline & & & 2025 & 2050 \\
\hline JAD1-4 & $\begin{array}{l}\text { Zone } 1(87,000 \text { dunum } \\
\text { in 2010) }\end{array}$ & $81,841.523$ & $103,596.865$ & $103,596.865$ \\
\hline JAD5-8 & $\begin{array}{l}\text { Zone } 2(74,959 \text { dunum } \\
\text { in 2010) }\end{array}$ & $71,803.344$ & $97,577.530$ & $104,489.941$ \\
\hline JAD9-16 & $\begin{array}{l}\text { Zone } 3(70,041 \text { dunum } \\
\text { in 2010) }\end{array}$ & $32,746.135$ & $55,733.922$ & $60,252.889$ \\
\hline \multicolumn{2}{|c|}{ Agriculture subtotal } & $186,391.003$ & $256,908.317$ & $268,339.95$ \\
\hline \multicolumn{2}{|c|}{ Total supply } & $268,630.723$ & $366,752.296$ & $420,294.799$ \\
\hline \multicolumn{2}{|c|}{ Total water resources Jordan LJRB } & 2010 & 2025 & 2050 \\
\hline \multicolumn{2}{|c|}{ Tiberias carrier pipe } & 47.00 & 100.00 & 100.00 \\
\hline \multicolumn{2}{|c|}{ Purchased DW to Amman } & 9.00 & 9.00 & 9.00 \\
\hline \multicolumn{2}{|c|}{ Groundwater wells in LJR Jordan } & 26.74 & 25.95 & 24.68 \\
\hline \multicolumn{2}{|c|}{ Yarmouk River } & 15.21 & 14.76 & 14.04 \\
\hline \multicolumn{2}{|c|}{$\begin{array}{l}\text { Import of treated WW (Irbid and } \\
\text { Amman) }\end{array}$} & 0.00 & 14.00 & 29.00 \\
\hline \multicolumn{2}{|c|}{ Mukheiba well field } & 27.70 & 26.88 & 25.57 \\
\hline Wadi Arab & Dam & 10.98 & 10.66 & 10.14 \\
\hline Wadi Zigl & ab Dam & 3.06 & 2.97 & 2.82 \\
\hline Wadi Al J & urum & 2.30 & 2.23 & 2.12 \\
\hline Wadi Abu & Ziad & 0.25 & 0.24 & 0.23 \\
\hline Wadi Yabi & is Diversion & 0.84 & 0.82 & 0.78 \\
\hline Wadi Kufr & & 2.43 & 2.36 & 2.24 \\
\hline Wadi Rajil & & 1.66 & 1.61 & 1.53 \\
\hline Zarqa carr & rier 1/King Talal Dam & 52.95 & 52.95 & 52.95 \\
\hline
\end{tabular}


Table 5.4 (continued)

\begin{tabular}{l|l|l|l}
\hline Zarqa carrier 2/King Talal Dam & 50.00 & 50.00 & 50.00 \\
\hline Shouib Dam & 6.25 & 6.07 & 5.77 \\
\hline Kafreen Dam & 11.51 & 11.17 & 10.62 \\
\hline Wadi Hisban & 0.78 & 0.76 & 0.72 \\
\hline Local WW reuse in LJR Jordan & 0.00 & 11.94 & 33.25 \\
\hline $\begin{array}{l}\text { WW reuse in LJR from } \\
\text { Amman/Northern GV }\end{array}$ & 0.00 & 22.40 & 44.80 \\
\hline Total (MCM/year) & 268.66 & 366.75 & 420.27 \\
\hline Deficit & 89.84 & 19.35 & 7.95
\end{tabular}

Table 5.5 Israeli water demands and supply in the Jordan valley

\begin{tabular}{|c|c|c|c|}
\hline $\begin{array}{l}\text { Total domestic water } \\
\text { demands Israel (CM/year) }\end{array}$ & 2010 & 2025 & 2050 \\
\hline Emek Hayarden & 990.000 & $1,166.880$ & $1,584.000$ \\
\hline Emek Hamaayanot & 990.000 & $1,166.880$ & $1,584.000$ \\
\hline Beit She'an & $1,530.000$ & $1,803.360$ & $2,448.000$ \\
\hline Hagilboa & 900.000 & $1,060.800$ & $1,440.000$ \\
\hline Total & $4,410.000$ & $5,197.920$ & $7,056.000$ \\
\hline \multirow{2}{*}{$\begin{array}{l}\text { Total agricultural water } \\
\text { demands Israel }\end{array}$} & \multirow[t]{2}{*}{2010} & \multicolumn{2}{|l|}{ CM/year } \\
\hline & & 2025 & 2050 \\
\hline Jordan valley WA & $21,237.000$ & $21,237.000$ & $21,237.000$ \\
\hline Afikey main WA & $52,015.000$ & $52,015.000$ & $52,015.000$ \\
\hline Harod WA & $22,000.000$ & $22,000.000$ & $22,000.000$ \\
\hline Fish ponds & $56,400.000$ & $56,400.000$ & $56,400.000$ \\
\hline Total & $151,652.000$ & $151,652.000$ & $151,652.000$ \\
\hline Total water demands & $156,062.000$ & $156,849.920$ & $158,708.000$ \\
\hline $\begin{array}{l}\text { Total water resources } \\
\text { Israel LJRB }\end{array}$ & 2010 & 2025 & 2050 \\
\hline $\begin{array}{l}\text { Groundwater wells (NE } \\
\text { mountain aquifer) }\end{array}$ & 22.00 & 20.00 & 18.00 \\
\hline $\begin{array}{l}\text { Lake of Tiberias/Jordan } \\
\text { river }\end{array}$ & 45.06 & 45.77 & 50.00 \\
\hline $\begin{array}{l}\text { Local wells (LJR basin) } \\
\text { Israel }\end{array}$ & 0.00 & 0.00 & 0.00 \\
\hline Harod stream & 54.00 & 46.00 & 32.00 \\
\hline Tavor stream & 2.00 & 2.00 & 2.00 \\
\hline LJR valley springs & 23.00 & 21.00 & 18.00 \\
\hline $\begin{array}{l}\text { Wastewater reuse in LJR } \\
\text { Israel }\end{array}$ & 2.82 & 3.33 & 4.52 \\
\hline $\begin{array}{l}\text { Wastewater reuse from } \\
\text { outside sources }\end{array}$ & 0.00 & 0.00 & 14.19 \\
\hline $\begin{array}{l}\text { Fish ponds reuse for } \\
\text { agriculture in LJR }\end{array}$ & 7.00 & 18.00 & 20.00 \\
\hline Total (MCM/year) & 155.88 & 156.10 & 158.71 \\
\hline Deficit (MCM/year) & -0.18 & -0.75 & 0.00 \\
\hline
\end{tabular}

pollution sources in the Israeli stretch from the Sea of Galilee to Naharyim is already foreseen by 2017 and in the stretch from Naharyim to the Harod Stream by 2020.
- By 2050 Jordan will use the Jordan River as its main water conveyor for water supply purposes instead of the current King Abdullah Canal. This implies that Jordan would stop diverting water from the Yarmouk and other tributaries into the $\mathrm{KAC}$, and instead diverts this water to the Jordan River to the possible extent.

- By 2050 Amman will not only receive 60 MCM/year from the Jordan Valley, as today, but will return an additional $60 \mathrm{MCM}$ of treated wastewater back to the Jordan Valley.

- The river has a natural tendency to become increasingly saline in southern direction, mainly due to brackish groundwater inflow near the Dead Sea. This implies that fresh water can only be supplied from the upper stretch of the river and more brackish water from the lower stretch of the river. Quality requirements for different types of consumption are the following:

- Raw drinking water quality $-<400 \mathrm{mg} / \mathrm{l}$

- Low Salinity/Semi Fresh irrigation water quality$<600 \mathrm{mg} / \mathrm{l}$

- Dates irrigation water quality $-<1500 \mathrm{mg} / \mathrm{l}$

- In 2050 Palestine will receive from the Jordan River totally $50 \mathrm{MCM} / \mathrm{year}$.

- In 2050 an additional $50 \mathrm{MCM} / \mathrm{year}$ of treated wastewater from the West Bank/East Jerusalem will be diverted into the Palestinian section of the Jordan Valley.

- Climate change will result by 2050 in a linear decrease of $20 \%$ of all water sources and increase in evaporation by $8 \%$.

\subsubsection{Salinity}

In the WEAP model, salinity is the only indicator of water quality. Designated salinity values of water sources are mentioned below and are documented in the model itself. The calculations of Chloride $(\mathrm{Cl})$ concentrations in the different reaches are based on simple mass balance with no decay mechanisms: Salinity of all the water sources is fixed throughout the year, except for the springs that nourish the Saline Water Carrier (SWC):

- Particularly, the salinity of the SoG does not change with water level and is fixed at $280 \mathrm{mg} / \mathrm{l}$;

- Runoff salinity is $50 \mathrm{mg} / \mathrm{l}$;

- Salinity of return flow from irrigation is 800 and $1500 \mathrm{mg} / \mathrm{l}$ for fresh and saline water respectively;

- Salinity of Israeli Sewage is $350 \mathrm{mg} / \mathrm{l}$;

- Effect of evaporation on salinity in the Lower part of the Jordan River itself is neglected. 
Table 5.6 Palestinian water demands and supply in the Jordan valley

Total domestic water demands palestine (CM/year) 2010 2025 2050

Palestinians

\begin{tabular}{|c|c|c|c|c|}
\hline PMD 1 & Bardala cluster MD & 315.540 & $1,184.575$ & $5,649.520$ \\
\hline PMD 2 & Al-Bassariya cluster MD & 273.840 & $1,105.090$ & $5,470.400$ \\
\hline PMD 3 & Al-Jiftlik cluster ND & 389.940 & $1,326.325$ & $5,969.200$ \\
\hline PMD 4 & Fasayil cluster MD & 69.420 & 715.575 & $4,592.080$ \\
\hline PMD 5 & Al-Auja cluster MD & 265.380 & $1,088.990$ & $5,434.000$ \\
\hline PMD 6 & Jericho MD & $2,046.720$ & $4,483.360$ & $13,087.280$ \\
\hline \multicolumn{2}{|c|}{ Subtotal Palestinian } & $3,360.840$ & $9,903.915$ & $40,202.480$ \\
\hline \multicolumn{5}{|c|}{ Settlements } \\
\hline IMD 1 & Cluster North ND & 128.250 & 0 & 0 \\
\hline IMD 2 & Cluster Central MD & 356.400 & 0 & 0 \\
\hline IMD 3 & Cluster South ND & 77.400 & 0 & 0 \\
\hline \multicolumn{2}{|c|}{ Subtotal Israeli settlers } & 562.050 & 0 & 0 \\
\hline \multicolumn{2}{|l|}{ Total } & $3,922.890$ & $9,903.915$ & $40,202.480$ \\
\hline \multirow{2}{*}{\multicolumn{2}{|c|}{ Total agricultural water demands Palestine }} & \multirow[t]{2}{*}{2010} & \multicolumn{2}{|l|}{ CM/year } \\
\hline & & & 2025 & 2050 \\
\hline \multicolumn{5}{|c|}{ Palestinians } \\
\hline PAD 1 & Bardala Cluster & $10,558.755$ & $13,658.850$ & $13,658.850$ \\
\hline PAD 2 & Al-Bassariya Cluster & $5,240.855$ & $14,396.297$ & $14,396.297$ \\
\hline PAD 3 & Al-Jiftlik Cluster & $5,400.437$ & $24,555.879$ & $24,555.879$ \\
\hline PAD 4 & Fasayil Cluster & $1,173.919$ & $20,329.361$ & $20,329.361$ \\
\hline PAD 5 & AI-Auja Cluster & $3,991.597$ & $23,147.039$ & $23,147.039$ \\
\hline PAD 6 & Jericho & $11,082.381$ & $29,083.044$ & $29,083.044$ \\
\hline \multicolumn{5}{|c|}{ Settlements } \\
\hline IAD 1 & Cluster North AD & $3,100.095$ & 0 & 0 \\
\hline IAD 2 & Cluster Central AD & $36,621.768$ & 0 & 0 \\
\hline IAD 3 & Cluster South AD & $8,000.662$ & 0 & 0 \\
\hline \multicolumn{2}{|c|}{ Palestine total } & $85,170.469$ & $125,170.470$ & $125,170.470$ \\
\hline \multicolumn{2}{|c|}{ Grand total water demands } & $89,093.359$ & $135,074.385$ & $165,372.950$ \\
\hline \multicolumn{2}{|c|}{ Total water resources Palestine LJRB } & 2010 & 2025 & 2050 \\
\hline \multicolumn{2}{|c|}{ Local wells (LJR) Palestine } & 32.00 & 63.11 & 46.64 \\
\hline \multicolumn{2}{|c|}{ Water import (Mekorot) } & 29.46 & 0.00 & 0.00 \\
\hline \multicolumn{2}{|c|}{ Local springs (Fara, Auja, Jericho, Pazael) } & 19.00 & 25.00 & 25.00 \\
\hline \multicolumn{2}{|c|}{ WW reuse Import from west bank } & 0.00 & 17.00 & 17.00 \\
\hline \multicolumn{2}{|c|}{ West bank floods } & 1.00 & 1.00 & 1.00 \\
\hline \multicolumn{2}{|c|}{ Jordan river (agriculture) Palestine } & 0.00 & 25.00 & 50.00 \\
\hline \multicolumn{2}{|c|}{ Wastewater reuse in LJR Palestine } & 0.00 & 3.96 & 25.73 \\
\hline \multicolumn{2}{|c|}{ Total (MCM/year) } & 81.46 & 135.07 & 165.37 \\
\hline \multicolumn{2}{|c|}{ Deficit } & 7.63 & 0.00 & 0.00 \\
\hline
\end{tabular}

\subsubsection{Groundwater Contribution}

\section{Groundwater Israel}

Direct contribution of groundwater to the LJR from Israel (north of Bezeq Stream) was calculated according to Holtzman, who quantified groundwater in two segments of the LJR, between the Yarmouk and Harod Stream. The model simulates groundwater contribution, by adding groundwater inflow in two reaches: below the Yarmouk and below Issachar. The annual contribution of groundwater into the LJR was estimated to be $18 \mathrm{MCM}$, with an average salinity of $1150 \mathrm{mg} / \mathrm{l}$. 
Table 5.7 Summary of proposed water resources to meet the projected water demands (MCM)

\begin{tabular}{|c|c|c|c|}
\hline Total water resources total LJR basin & 2010 & 2025 & 2050 \\
\hline Tiberias Carrier Pipe to Jordan & 47.00 & 100.00 & 100.00 \\
\hline Purchased DW to Amman & 9.00 & 9.00 & 9.00 \\
\hline Groundwater Wells in LJR Jordan & 26.74 & 25.95 & 24.68 \\
\hline Yarmouk River & 15.21 & 14.76 & 14.04 \\
\hline Import of Treated WW (Irbid and Amman) & 0.00 & 14.00 & 29.00 \\
\hline Mukheiba Well Field & 27.70 & 26.88 & 25.57 \\
\hline Wadi Arab Dam & 10.98 & 10.66 & 10.14 \\
\hline Wadi Ziglab Dam & 3.06 & 2.97 & 2.82 \\
\hline Wadi Al Jurum & 2.30 & 2.23 & 2.12 \\
\hline Wadi Abu Ziad & 0.25 & 0.24 & 0.23 \\
\hline Wadi Yabis Diversion & 0.84 & 0.82 & 0.78 \\
\hline Wadi Kufrina & 2.43 & 2.36 & 2.24 \\
\hline Wadi Rajib & 1.66 & 1.61 & 1.53 \\
\hline Zarqa Carrrier 1/King Talal Dam & 52.95 & 52.95 & 52.95 \\
\hline Zarqa Carrier 2/King Talal Dam & 50.00 & 50.00 & 50.00 \\
\hline Shouib Dam & 6.25 & 6.07 & 5.77 \\
\hline Kafreen Dam & 11.51 & 11.17 & 10.62 \\
\hline Wadi Hisban & 0.78 & 0.76 & 0.72 \\
\hline Wastewater Reuse in LJR Jordan & 0.00 & 11.94 & 33.25 \\
\hline Wastewater Reuse from Jordan & 0.00 & 22.40 & 44.80 \\
\hline Groundwater wells (NE mountain aquifer) & 22.00 & 20.00 & 18.00 \\
\hline Lake of Tiberias/Jordan River & 45.06 & 45.77 & 50.00 \\
\hline Harod Stream & 54.00 & 46.00 & 32.00 \\
\hline Tavor Stream & 2.00 & 2.00 & 2.00 \\
\hline LJR Valley Springs & 23.00 & 21.00 & 18.00 \\
\hline Wastewater Reuse in LJR Israel & 0.00 & 2.08 & 4.52 \\
\hline Wastewater Reuse from Israel & 0.00 & 0.00 & 14.19 \\
\hline Fish Ponds Reuse for Agriculture in LJR & 10.00 & 20.00 & 20.00 \\
\hline Local Wells (LJR) Palestine & 32.00 & 63.11 & 46.64 \\
\hline Water Import (Mekorot) & 29.46 & 0.00 & 0.00 \\
\hline Local Springs (Fara, Auja, Jericho, Pazael) & 19.00 & 25.00 & 25.00 \\
\hline WW Reuse Import from West Bank & 0.00 & 17.00 & 17.00 \\
\hline West Bank Floods & 1.00 & 1,00 & 1,00 \\
\hline Jordan River (agriculture) Palestine & 0.00 & 25.00 & 50.00 \\
\hline Wastewater reuse in LJR Palestine & 0.00 & 3.96 & 25.73 \\
\hline Grand total & 506.18 & 658.67 & 744.34 \\
\hline
\end{tabular}

*Part of these resources are brackish, and can only be reused for specific purposes

\section{Groundwater Palestine}

For the West Bank (south to Bezeq stream) the current WEAP model assumes that groundwater inflow is constant throughout the year and is about 5-6 MCM/month. The salinity levels have been assumed to be similar range as measured by Farber et al.

\section{Groundwater Jordan}

In the southern part of East Bank, the shallow groundwater system consists of lacustrine sediments and Clastic fluvial components. The aquifer has been developed largely since the 1960s, and many shallow wells have been drilled, largely for irrigation purposes. Consequently, groundwater levels have dropped and salinity levels increased substantially. Where historically groundwater flow in the Eastern Jordanian valley area had a westwards direction, today more water is abstracted that recharged naturally. In this model it has therefore been assumed that there is no annual contribution of groundwater into the LJR from the southern Jordanian side.

\subsubsection{Water Supply Assumptions}

\section{WEAP Modeling Assumptions}

The aim of the Full Cooperation Scenario is to turn the LJR into the main water conveyor in the Jordan valley, turning the river into a multiuse water body, where its ecological integrity is maintained.

The following conditions were assumed:

- Urban and domestic water will be provided mainly by local wells, springs and dams to ensure water quality standards and reliability.

- Agricultural water will be provided through the reuse of treated wastewater, through the King Abdullah Canal and West Ghor pipeline on the short term; and through the Jordan River on the long term.

- Israel will substantially reduce pumping water from the Sea of Galilee to the National Water Carrier.

- Israel will maintain present agricultural consumption in the valley.

- By 2025 Jordan will stop diverting water from the Yarmouk and other tributaries to the KAC and have started using the LJR as main conveyor instead, to the possible extent.

- By 2050 Palestinian irrigation requirements will be supplied through wastewater reuse from within the Jordan Valley and from outside towns such as Ramallah and Nablus (totally about $43 \mathrm{MCM} / \mathrm{year}$ ), and from the Jordan River (50 MCM/year) and from local water resources (32 MCM/year).

- Amman will consume $100 \mathrm{MCM} / \mathrm{year}$ of potable water from the KAC and provide some $80 \mathrm{MCM} / \mathrm{year}$ of effluents (of which $70 \%$ will be used in the LJV).

- Irbid will produce $13 \mathrm{MCM} / y e a r$ of effluents, of which some $9 \mathrm{MCM} /$ year will be used in the LJV.

- Water demands are in accordance with the above presented tables. 
Table 5.8 Anticipated flows in the lower part of the Jordan river (2050)

\begin{tabular}{l|l|l|l|l|l}
\hline Location on LJR & $\begin{array}{l}\text { MCM } \\
\text { month min }\end{array}$ & $\begin{array}{l}\text { MCM } \\
\text { month max }\end{array}$ & $\begin{array}{l}\text { Avg } \\
\text { mg/l Cl }\end{array}$ & $\begin{array}{l}\text { Calculated* } \\
\text { MCM/year }\end{array}$ & $\begin{array}{l}\text { Estimated** MCM/year (incl additional 210 MCM } \\
\text { reuse inflow from wider region) }\end{array}$ \\
\hline Alumot & 17 & 27 & 280 & 238 & 238 \\
\hline Yarmouk inflow & 17 & 33 & 265 & 274 & 274 \\
\hline Withdrawal to KAC & 18 & 35 & 320 & 291 & 291 \\
\hline $\begin{array}{l}\text { Withdrawal to } \\
\text { Tirza/JAD2 }\end{array}$ & 4 & 21 & $700^{\mathrm{a}}$ & 138 & 230 \\
\hline $\begin{array}{l}\text { Withdrawal to } \\
\text { Palestinian dates }\end{array}$ & 2 & 16 & $1350^{\mathrm{b}}$ & 105 & 238 \\
\hline $\begin{array}{l}\text { After last } \\
\text { withdrawal }\end{array}$ & 0 & 10 & 1350 & 55 & 215 \\
\hline \begin{tabular}{l} 
After brine inflow \\
\hline $\begin{array}{l}\text { Outflow into the } \\
\text { dead sea }\end{array}$
\end{tabular} & 3 & 14 & 3050 & 90 & 287 \\
\hline
\end{tabular}

*Major inflows into the LJR that are above $5 \mathrm{MCM} / \mathrm{year}$ are the following:

Sea of Galilee 238 MCM/year; Groundwater inflow, spread along the entire river 45 MCM/year; Western Brine Carrier (inflow at Wadi Qelt) 35 MCM/year; Yarmouk 34 MCM/year; Valley of Springs 12 MCM/year; Harod Stream 8 MCM/year; Wadi Arab 8 MCM/year

**Assuming additional $197 \mathrm{MCM} /$ year of treated wastewater diverted into the river from the wider regions in Jordan, Israel and Palestine, possibly generating hydropower at the same time

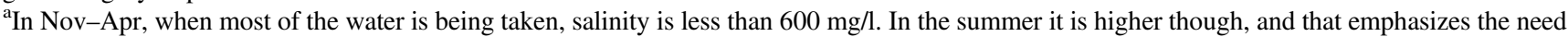
for large reservoirs to facilitate more extraction in winter and also dilution of water

${ }^{\mathrm{b}}$ In summer and autumn salinity can top $1500 \mathrm{mg} / \mathrm{l}$, which again necessitates reservoirs

- Sea of Galilee is to be kept on a medium water level between the top and bottom rend lines (What is called now the "green line" by the IWA).

- All pollution from the river, with possible exception of fishponds groundwater leakage, will be removed from the LJR by 2025 .

- All locally produced municipal wastewater will be treated and reused for irrigation.

- Water quality demand with respect to $\mathrm{mg} / \mathrm{l}$ of Chlorides is:

- Drinking water- $400 \mathrm{mg} / \mathrm{l}$

- Fresh irrigation $-600 \mathrm{mg} / \mathrm{l}$

- Dates irrigation-1500 mg/l

- Water sources in the west bank amount to $85 \mathrm{MCM} / y e a r$ which include the Eastern mountain aquifer and springs.

- Climate change will result by 2050 in a linear decrease of $20 \%$ of all water sources and increase evaporation by $8 \%$.

\section{Main Water Supply Assumptions}

Within WEAP model run for 2050 the LJR will be largely divided into 4 zones. The following assumptions have been made:

- Water of drinking quality within the valley will be supplied from the Sea of Galilee to upstream of the confluence with Harod.

- Water for fresh irrigation quality purposes will be provided from the confluence with Harod to upstream of the confluence with Zarqa.
- Water for Dates irrigation quality purposes will be provided from the confluence with Zarqa to the confluence with Wadi Qilt.

- From the Confluence with Wadi Qilt to the Dead Sea the river water quality will not be rehabilitated.

The brine water resources in the valley will be conveyed to the lower stretch of the Jordan River, at the confluence with Wadi Qilt and from there through the river into the Dead Sea. At this point the river will receive brine from two sources:

- Western Brine Carrier-A new conveyor west to the LJR that will carry water of the Saline Water Carrier, brine originating from desalination, and from fishponds discharges.

- Brine from the Abu Zeighan desalination plant.

In addition, three major pumping points will be established as follows:

\section{Pumping To the KAC}

Pumping to the KAC upstream the confluence with Harod - The KAC will be used from this point on as a conveyor of drinking water quality to Jordan. It will convey 170 $\mathrm{MCM} /$ year from the LJR, of which $70 \mathrm{MCM}$ will be supplied to JAD1 (irrigation), $22 \mathrm{MCM}$ to JMD 1 (municipal) and $70 \mathrm{MCM}$ will be transferred southwards. A reservoir network with a capacity of $30 \mathrm{MCM}$ will be built to support supply to JAD1 and facilitate storage from winter to 
summer. The rest of the water to JAD1 will come from Mukheiba well and treated WW from JMD1 and Irbid.

\section{Pumping for Irrigation at Zarqa}

The water pumped upstream of the confluence with Zarqa for irrigation purposes will be distributed as follows:

12 MCM to a network of Palestinian reservoirs with a storage capacity of $40 \mathrm{MCM}$. The backbone of the system will be the existing Tirza reservoirs that will now serve for fresh irrigation. The reservoirs will also receive $32 \mathrm{MCM}$ of treated local wastewater, $32 \mathrm{MCM}$ of effluents from eastern Jerusalem, 18 MCM from local aquifer/springs and $1 \mathrm{MCM}$ of floods from Wadi Fara'a to sustain Palestinian agriculture

$60 \mathrm{MCM}$ to Jordan as follows: (1) $55 \mathrm{MCM}$ to JAD2. A reservoir network of $20 \mathrm{MCM}$ will be built to support supply to JAD2 and facilitate storage from winter to summer. JAD2 will also receive $9 \mathrm{MCM}$ of effluents from JMD2; (2) 5 MCM to JAD3. The bulk of supply to JAD3 will come as 55 MCM of treated WW from JMD3 and Amman. For that a reservoir of $10 \mathrm{MCM}$ will be required.

Pumping from the Jordan River

The pumping of $50 \mathrm{MCM} / \mathrm{year}$ from the Jordan River for Palestinian agriculture in the west bank will be concentrated in the winter so a network of reservoirs with a capacity of 40 MCM will have to be built.

\subsubsection{Impacts on Flows in the Jordan River}

The presented data and assumptions lead to the above flow regime of the Lower part of the Jordan River.

The calculated water balance will provide all demands in the valley by 2050 , and the related flow in the Jordan River will reach in maximum just before the KAC withdrawal, with $291 \mathrm{MCM} / \mathrm{year}$. Next it will reduce towards the Dead Sea $(90 \mathrm{MCM} / \mathrm{year}$ outflow into the Dead Sea). If one would aim at an outflow of $300 \mathrm{MCM} /$ year into the Dead Sea instead, this would require an additional inflow of 210 $\mathrm{MCM} /$ year, including for instance a future contribution of Syria of $100 \mathrm{MCM} / \mathrm{year}$ and additional $110 \mathrm{MCM} / \mathrm{year}$ of inflow into the valley from wider sources of treated wastewater.

Detailed assessment of these alternative and additional resources goes beyond the scope of this study. However, in line with earlier studies, including those of the World Bank Study on Alternatives related to the Red-Dead Sea Water Conveyance Project, it may be assumed that this water can be identified in 2050 as inflow of additional treated wastewater into the valley from the wider regions in Jordan, Israel and Palestine.

Such additional release of water into the Dead Sea will come at a certain cost, which may be directly compared to economic benefits of the Dead Sea economy by 2050. It should be noted though that flows required for stabilizing the
Dead Sea Water levels are substantially higher than 400 $\mathrm{MCM} / \mathrm{year}$, and may reach to more than $800 \mathrm{MCM} / \mathrm{year}$. These could be met according to WEDO/EcoPeace if industry in the south of the Dead Sea were to replace their evaporation ponds with the use of membrane technology to extract Dead Sea minerals.

The Palestinian fresh irrigation demands will be met from treated wastewater (32 MCM locally produced and the rest imported from upper Palestine. Here to, a reservoir network of $35 \mathrm{MCM}$ will be required as wastewater supply is constant but the agricultural demand fluctuates. The backbone of the system will be the existing Tirza reservoirs that will now serve for fresh irrigation. The existing pumps on the Jordan River to Tirza reservoirs will serve as backup, but will not be used regularly.

The above water balance relies on natural resources only. Future water demands however, can be met, on average, via optimal usage of natural sources. Meeting the demand will become increasingly difficult though, with water quality problems throughout summer and autumn. For that reason large reservoirs (with a total capacity of nearly $150 \mathrm{MCM}$ on both sides of the river) may be considered to facilitate storage from winter to summer. Alternatively part of this storage requirement might be realized through groundwater recharge and storage facilities. This would lead to less evaporation, and would require detailed hydrogeological assessments in terms of availability and suitability of shallow aquifer systems.

The above is true for average and wet years only. In dry years, which are frequent in this region, local water and WW will not be able to quench the demand. The $150 \mathrm{MCM}$ of reservoirs will not mitigate shortage in dry years, as that storage volume is seasonal, from winter to summer, and not annual. There is little point in adding even more storage capacity as consecutive dry years are common and thus, water from wet years cannot be stored for future dry years (much less considering evaporation).

Hence, the solution is either reducing demand, or relying on a stable external water source. Calculations show that additional 120-190 MCM/year from an external source will be required to meet the environmental goals set by WEDO/EcoPeace, on average. In dry years, the environmental goals will not be met in full though. The added water will also enable reliable water supply for consumers across the valley, except for the most extreme dry years. ${ }^{1}$ That

\footnotetext{
${ }^{1} 2014$ was the driest year in recorded history, to the extent that the natural water balance of the Sea of Galilee was negative, meaning that even if no water would have been pumped from the lake, its level would still go down that year. In such an extreme case, the Sea of Galilee cannot sustain a release of $250 \mathrm{MCM}$ and so, water will be in short supply. Such a year however is infrequent.
} 
water will also allow reducing the required storage capacity, as more water will be pumped in the summer, when it is needed most.

Various alternative measures from outside the study area may be considered in this respect, such as presented in the World Bank Study on Alternatives for the Read-Dead Sea Program, Sept 2012.

1. Additional sources of generated wastewater from regions outside the Jordan Valley, but still located within the watershed area may be treated and conveyed to the Jordan Valley for agricultural applications.

2. The agricultural water demands in the Jordan Valley may be further reduced through water efficiency measures and/or by limiting certain lower priority agricultural activities. This may lead to a reduction of the total agricultural demands in the study area from 557 $\mathrm{MCM} /$ year as assumed above, to $490 \mathrm{MCM} / \mathrm{year}$ instead.

3. Replacing the $60 \mathrm{MCM} / \mathrm{year}$ supply of water from the Jordan Valley to Amman by other water sources or measures, such a Red Sea desalination, increasing DISI aquifer supply; maximization of wastewater reuse within the urban context, or lowering unaccounted for water percentages within the city.

4. Conveying desalinated water from the Mediterranean to the upper part of the Jordan Valley.

5. Or a combination of above four measures.

\subsection{Environmental Flows by 2050}

\subsubsection{Introduction}

Rehabilitation of the ecological values connected to the Jordan River depends not only on restoring good quality water and protection against external pollution sources, but also on a flow regime in the river that sustains the ecological water needs various seasons.

The concept of environmental flows includes setting conditions for an average flow along different sections of the river, and on conditions related to floods and droughts. For instance, during extreme low flows, native species may out-compete exotic species that have not adapted to these circumstances, while during periods of more stable low flows, feeding and spawning activities of fish and recreational activities may be supported. On the other hand, minor floods may prevent vegetation from invading river channels and can wash out plants, delivering large amounts of sediment and organic matter downstream in the process, while large floods may even change the flow path of a river and create flood plains that provide new nutrient-rich niches for certain habitats. One can predict that the more a flow event deviates from the average annual flow, in terms of floods or drought intensities, the rarer it will be.

Defining the optimized environmental flow condition for the Jordan River is subject to the specific ambitions one has in terms of ecological restoration, and remains subjective to the extent that certain flow regimes may be beneficial for specific species and be less beneficial for others.

However, the substantial reduction of flow in the Jordan River since the 1950 s resulted in a narrower and more canalized river ecosystem. Less water resulted in much slower velocities, reducing the habitats depending of flows, such as falls, cascades and rapids. Smaller flows also mean less dilution with inflowing polluted water, such as brackish (ground) water or wastewater. This resulted in higher pollution concentrations in the river stream. As a result, the ecology of the Jordan River is now reduced to pockets of high resistant and medium to slow velocity habitats. Reduction in water flows, but also dams in the river and its tributaries, resulted also in smaller river's sediment loads. Slower velocities carry far less sediment with smaller grain sizes. The formation of streamside water bodies, such as deserted meanders, has stopped, and related habitats have disappeared from the river's ecosystem.

If healthy freshwater ecosystems are to be restored, it is important to address the quality of the water; the seasonable fluctuation of the flow; the frequency and the duration and variability of floods and droughts.

\subsubsection{Environmental Flow Alternatives}

In May 2010, WEDO/EcoPeace issued a report called: "Towards a Living Jordan River: An Environmental Flows Report on the Rehabilitation of the Lower part of the Jordan River". This report presents four alternatives strategies for restoration of viable environmental flows in the Lower part of the Jordan River and related ecological values of the river system:

(1) Full Ecological Restoration;

(2) Partial Restoration;

(3) River Rehabilitation;

(4) Flow Enhancement.

\section{"Full Restoration" Alternative}

Under this strategy, the pollution sources into the Jordan River are to be removed, including treatment of all wastewater generated in the valley and saline water from the Saline Water Carrier. The salinity of the water in the Jordan River shall not exceed $250 \mathrm{ppm}$ in the winter and $350 \mathrm{ppm}$ in summer and in the southern section it should not exceed 
$750 \mathrm{ppm}$. The saline water of the Sea of Galilee's salty springs shall be diverted away from the Jordan River, for instance through desalinization and removal of its brine from the Jordan Valley.

Full restoration would also mean that the original pre-1950 flows are to be restored to $1200-1400 \mathrm{MCM} / \mathrm{year}$. This very ambitious objective implies that for instance 500 $600 \mathrm{MCM}$ is to be released extra from the Sea of Galilee into the Jordan River, and approximately 500-600 MCM/year from the Yarmouk River. This Full ecological restoration strategy also requires at least 3 minor floods (c.a. $20-50 \mathrm{~m}^{3}$ / s) per year, to be achieved for instance by fully opening the dams for $24 \mathrm{~h}$, three times every winter and 1 major flood (c. a. $200 \mathrm{~m}^{3} / \mathrm{s}$ ) every 3 years. In order to bring back the original habitats of the river, also the shape and flow path of the river is to be restored, including reconstruction of meanders, cascades and waterfalls. Clearly this very ambitious strategy would require high investments, a revolutionary change in the water regimes of particularly Israel and Jordan, and would be globally the first full river restoration in its kind. This strategy will lead to recovery of a healthy water related eco system comparable to the historic situation of the area.

"Partial Restoration" Alternative

Partial restoration of the river is defined here as removal of the pollution sources into the Jordan River, including treatment of all wastewater generated in the valley, and dilution of the saline water in the Jordan River from the Saline Water Carrier with fresh water, so that the water in the Jordan River shall not exceed 500 ppm in the winter and $750 \mathrm{ppm}$ in summer and in the southern section it should not exceed 1,500 ppm. For this purpose the saline water of the Sea of Galilee's salty springs could for instance be mixed with fresh water originating from the Sea of Galilee and the Yarmouk River.

Partial restoration is also defined here as generating flows of 600-800 MCM/year. This ecological restoration strategy also requires at least one minor flood (c.a. $20-50 \mathrm{~m}^{3} / \mathrm{s}$ ) per year, to be achieved for instance by fully opening the dams for $24 \mathrm{~h}$ every winter. In order to bring back the original habitats of the river, also the flow bed of the river is to be widened to 50-70 $\mathrm{m}$ in the north and $25-40 \mathrm{~m}$ in the south, with flood plains on both sides. New meanders, cascades and waterfalls are to be constructed to some extent. This strategy would require considerable investments and a substantial change in the water regimes and national water policies of particularly Israel and Jordan. This strategy will lead to recovery of healthy water related eco systems.

"River Rehabilitation" Alternative

The river rehabilitation strategy is less ambitious than the two strategies described above. It is defined as full treatment of all wastewater generated in the valley, and allowing discharge of treated wastewater into the Jordan River to maximum $25 \%$ of the river's base flow. The water in the Jordan River shall not exceed $750 \mathrm{ppm}$.

River Rehabilitation is also defined here as generating flows of 400-600 MCM/year. This ecological restoration strategy requires again at least one minor flood (c.a. 20-50 $\mathrm{m}^{3} / \mathrm{s}$ ) per 2 years, to be achieved for instance by fully opening the dams for $24 \mathrm{~h}$ every other winter. In order to bring back the original habitats of the river, also the flow bed of the river is to be widened to $50-70 \mathrm{~m}$ in the north and 15$30 \mathrm{~m}$ in the south, with flood plains on both sides. New meanders, cascades and waterfalls are to be constructed to some extent. This strategy would require investments and a substantial change in the water regimes of particularly Israel and Jordan. This strategy will lead to significant recovery of the water related eco systems.

\section{"Flow Enhancement" Alternative}

The Flow Enhancement strategy is defined as enhancing the base flow of the Jordan River only the basis of treating all domestic and fishpond related wastewater, and discharging the treated effluent into the Jordan River, without depending on additional release from the Sea of Galilee or the Yarmouk River. Under this strategy the saline water carrier would continue to flow into the Jordan River, leaving the salinity levels at $3000 \mathrm{ppm}$ in the winter and $4000 \mathrm{ppm}$ in summer. In the southern section is should not exceed $10,000 \mathrm{ppm}$, which is $1 \%$ salt content.

The Flow enhancement is also defined as generating flows of 300-400 MCM/year, to be generated all from treated wastewater. This implies that all generated wastewater shall be treated and discharged into the river, without being reused for agricultural or other purposes. On the other hand, this also implies that no additional water is required from the Sea of Galilee or the Yarmouk River. This ecological restoration strategy requires again at least one minor flood (c.a. $20-50 \mathrm{~m}^{3} / \mathrm{s}$ ) per 2 years, to be achieved for instance by fully opening the dams for $24 \mathrm{~h}$ every other winter. In order to bring back the original habitats of the river, also the flow bed of the river is to be widened to 50$70 \mathrm{~m}$ in the north and $15-30 \mathrm{~m}$ in the south, with flood plains on both sides. New meanders and cascades are to be constructed to some extent, but no waterfalls. This strategy would require a substantial change in specifically the water regimes in the valley itself. This strategy will lead to substantial, but restricted recovery of the water related eco systems.

\section{WEDO/EcoPeace's Preferred Alternative}

Based on an assessment of these alternatives and comments and feedback received by participants of the Study's National and Regional Advisory Committee, WEDO/EcoPeace recommends a flow release of approximately $100 \mathrm{~m}^{3} / \mathrm{s}$ from Alumot dam for a $24 \mathrm{~h}$ period (less than $9 \mathrm{MCM}$ ) to cause an initial flood to make a significant 
ecological difference without flooding surrounding properties. The release should take place during the winter months to simulate natural seasonal flooding and would act to flush fine sediment and pollutants and provide significant habitat improvement in the short term. According to WEDO/EcoPeace this "re-start" should then be followed by the allocation of fresh water resources to halt the river's continuing deterioration, leading to a water flow of 400 MCM annually, including one minor flood annually and a reduction of salinity levels to no more than $750 \mathrm{ppm}$.

\subsubsection{Environmental Flow Regime for $\mathbf{2} 050$}

Within the framework of this Master Plan the following Environmental Flow Strategy has been assumed. The 2050 Environmental Flow regime is based on a multi-functional river approach. This means that the river will be used for both ecological, economic/tourism and water conveyance purposes, as such maximizing the flow within the river itself, and maximizing the ecological/economic value of the water flow. This implies that all sources of pollution have been mitigated and that the river water will have reclaimed a natural water quality, only affected negatively by the natural salt inflow from the southern brackish aquifer systems.

In accordance with the study's water modeling calculations this will require that by 2050 some $238 \mathrm{MCM} / \mathrm{year}$ of water will be released into the Jordan River from the Sea of Galilee. In addition, the river will receive $12 \mathrm{MCM} / \mathrm{year}$ from the Valley of Springs, $8 \mathrm{MCM}$ /year from the Harod Spring and $8 \mathrm{MCM} /$ year from Wadi Arab. It will also receive $18 \mathrm{MCM} /$ year as natural groundwater outflow in the Israeli part of the valley, and 5-6 MCM/year in the Palestinian part of the valley. No groundwater contribution from the Jordanian side is foreseen. However, it is assumed that by 2050 Jordan will use the Jordan River instead of the King Abdullah Canal for conveying water from north to south to the point where brackish groundwater naturally infiltrates into the Jordan River, near the Harod Stream.

In addition it is proposed to have at least one minor flood (c. a. $20-50 \mathrm{~m}^{3} / \mathrm{s}$ ) per 2 years, to be achieved for instance by fully opening the Damya dams for 24 h every other winter. In order to bring back the original habitats of the river, also the flow bed of the river will be widened to $50 \mathrm{~m}$ in the north and at least 15 m down to Wadi Qelt, with flood plains on both sides. This includes construction of new meanders and cascades.

This measure will lead to an average flow of up to 300 $\mathrm{MCM} / \mathrm{year}$ in the upper section of the river, and around 100 $\mathrm{MCM} /$ year of water flowing into the Dead Sea. The average salt content will be between 300-700 ppm in the upper section and around $1350 \mathrm{ppm}$ in the lower section, which is highly acceptable from an ecological point of view. The final southern stretch of the river, below Wadi Qelt will see higher concentrations up to $3000 \mathrm{ppm}$ due to brackish groundwater seepage and discharge of the Saline Water Carrier.

In order to reach a minimum flow of at least $400 \mathrm{MCM}$ of fresh water per year throughout the major stretch of the Jordan River, one depends on an additional contribution of $100 \mathrm{MCM} / y e a r$ from Syria by 2050 through the Yarmouk River; and an additional inflow of $100 \mathrm{MCM} / \mathrm{year}$ of treated wastewater into the Jordan River from the wider region in Israel, Jordan and Palestine around the Jordan Valley. However, these options will come against certain costs of probably not below $30 \mathrm{MUSD} / \mathrm{yr}$, which is to be assessed in a detailed cost-benefit analysis, for instance during the feasibility assessment phase of such interventions.

In all, the model calculations show that a sustainable and environmentally friendly water regime can be created in the Jordan Valley by 2050 that creates a clean and healthy river system and facilitates the interests all riparian states appropriately, including the future independent state of Palestine.

\subsection{Sensitivity Analysis}

The above economic projections are subject to a series of assumptions as part of the 2050 Vision for the Jordan Valley. These assumptions include full realization of the three-state co-operation scenario. Palestine will become an independent state, and an equitable partner to both Israel and Jordan. It is assumed that the three together will have established the Jordan River Basin Management Organization, responsible for river basin management, overseeing the agreed water supply frameworks and jointly managing the Jordan River as the major natural and multi-functional surface water body in the region.

Another assumption is that the three parties will have agreed on a joint security management framework that ensures maximized security against external threats against all Israeli, Palestinian and Jordanian people living in and around the Jordan Valley. It is assumed that this security framework will be based on unbiased, professional and joint assessment of security risks, and mitigation plans that effectively address these risks.

Lack of meeting these conditions will have a direct negative impact on the socio-economic status of the Jordan Valley as projected above, including very limited economic development perspectives for the Palestinian people, and limited synergetic economic impacts for Israel and Jordan. In addition, most of the proposed regional interventions cannot be implemented, such as joint and optimized development of the Jordan River and joint development of the regional economy, including the tourism sector. This will lead to much lower economic development projections for 2050 , which will likely not very much exceed the economy under the current status quo. 
The above economic projections also assume that by 2020 all pollution sources will have been mitigated or removed from the Jordan Valley, including wastewater, saline water and solid waste related pollution sources. Furthermore, it is assumed that all regionally generated wastewater will be treated and reused for agricultural purposes.

Lack of meeting these conditions will have large impacts on the water economy in the valley. Ongoing pollution will lead to continuous contamination of the Jordan River, which will block all interventions related to sustainable development of the river, including tourism and economic development, ecological restoration of the river ecosystem, and using the river as natural water conveyor. Moreover, ongoing pollution will continue to be a threat for public health, particularly for the Jordanians and Palestinians, with related negative private investment and economic growth potentials. Not treating and reusing the locally generated wastewater will furthermore have a serious impact on the 2050 water balance, particularly for the agricultural sector. This Master Plan assumes that by 2050 the total agricultural water demands in the Jordan Valley will be around 752 $\mathrm{MCM} / \mathrm{year}$, of which about $142 \mathrm{MCM} / \mathrm{year}$, or $19 \%$ if provided through reuse of treated wastewater. A similar reduction in agricultural economic outputs may be expected if treatment and reuse of wastewater would not take place.

The majority of investments foreseen in the Jordan Valley relate to urban and infrastructure development, about 3.4 Billion USD, or $80 \%$ of the total investments. This package is crucial for reaching the economic growth assumed in this Master Plan, and to provide the Jordanian and Palestinian needs in terms of housing, roads, education, medical care and utilities. Moreover, these interventions will attract private investors necessary for creating the economic dynamics in the Jordan Valley, required for sustainable growth and prosperity.

If these investments will not materialize, much of the foundation under the economic growth projections presented in this Master Plan will be absent. The economic activities, including the construction, services and tourism sectors would not grow to the extent presented here, or may even shrink further. The total valley economy, depending on which negative scenarios will be adopted, would likely be stuck somewhere between 5-20 Billion USD in 2050, instead of the foreseen 73 Billion USD economy under this Master Plan. Moreover, the Palestinian economy and partly the Jordanian economy a well remain largely dependent on the international financial donor community for many years to come.

Climate change will likely have an important impact on the water balance in the Jordan Valley. This study's Baseline Report indicates that the northern part of the East Bank of the Jordan Valley (Jordan) will be impacted most negatively by climate change, with a foreseen substantial reduction of annual and winter rainfall, although the summer rainfall will increase slightly. The southern part of the East Bank will see a slight improvement of rainfall conditions, both annually as during the summer. The Western part of the Jordan Valley seems to be impacted to a smaller degree, and may also experience some positive impacts with slightly increasing rainfall gradually shifting southwards, both annually as well as during the winter period. The Jordan Valley in the lower part of the West Bank remains to be a very dry region. In addition, the evaporation rates will increase throughout the valley as result of increasing average annual temperatures, leading to more losses in terms of evaporation from surface waters like the Sea of Galilee, the Jordan River, reservoirs and open fishponds, and of more losses due to evapotranspiration by vegetation, mainly within the agricultural sector.

The Master Plan assumes that the average annual reduction in water resources due to Climate Change by 2050 will be $0.2 \%$, leading to an accumulated reduction of $7.7 \%$ of the locally generated water resources by 2050 compared to the year 2010 , or about $18 \mathrm{MCM} / \mathrm{year}$. The projected water balance presented in this Master Plan was calculated accordingly. However, if the climate impacts will be more severe, say around $15 \%$ reduction of locally generated water resources by 2050 , the system will experience an additional loss of $17 \mathrm{MCM} / \mathrm{year}$ by 2050 (or a total climate change inflicted loss of $35 \mathrm{MCM} / \mathrm{year}$ ) compared to 2010. If this extra loss would be fully at the expense of the agricultural sector the result is a reduction of $2.3 \%$ of agricultural outputs by 2050. If instead this extra loss is directly linked to the Jordan River flow, this will lead to a flow reduction of $8.5 \%$ in the upper reach of the Lower part of the Jordan River, and about $17 \%$ in the downstream section the Lower part of the Jordan River.

The current geopolitical context in and around the Jordan Valley today is complex. The Peace process between Israel and Palestine is still in a dead log, and the political turmoil in the neighbor countries, particularly in Syria and Iraq is strong. In august 2014 the United Nations had registered 619,000 official refugees from Syria in Jordan, with over 80,000 registered in the refugee camp Za'atri, located just outside the study area. In this Master Plan it has been assumed today a total foreign population of about 250,000 lives in the Jordanian part of the study area.

In this Master Plan it is assumed that by the Peace Process will be concluded positively, leading to an independent Palestinian State by 2020. By 2050 this will lead to close and effective co-operation among the three riparian states on all key issues related to joint economic development, water management and security management. In addition, this Master Plan assumes that in Jordan the high number of foreign inhabitants in the valley will gradually decline to 150,000 people by 2050 as a result of assumed improving 
geopolitical and economic conditions in their countries of origin, including Syria, Iraq and Egypt.

If the regional geopolitical context will not evolve towards this positive direction, one may expect that the number of foreign inhabitants in the valley will remain high. Assuming that the current numbers of 250,000 people will remain, this will lead to higher demands for instance in terms of household water supply (about $3 \mathrm{MCM} / \mathrm{year}$ extra compared to the projections in this Master Plan), and to higher demands in terms of public services and utilities.

High geopolitical uncertainties will also put more emphasis on the need for an effective and joint security shield against external threats. One may also raise the question whether more severe external geopolitical uncertainties would lead to more isolationistic behavior of the three riparian countries in the future, and therefore to reduce willingness to strive towards peace and regional co-operation. If so, this will clearly lead to direct negative impact on the socio-economic status of the Jordan Valley as projected here, including very limited synergetic economic impacts for the three riparian states.

In the vision for 2050 the Jordan River will play a crucial and multi-functional role. This implies that the river will serve different important functions at the same time, in terms of sustaining ecology, supporting tourism and related economic development, and conveying and supplying water throughout the valley. Without additional external sources, the flow in the Jordan River will reach a maximum of 291 $\mathrm{MCM} / \mathrm{year}$, and will be reduced to $90 \mathrm{MCM} / \mathrm{year}$ near the outflow into the Dead Sea by 2050. The average salt content will be between $300-700 \mathrm{ppm}$ in the upper section of the river and around $1350 \mathrm{ppm}$ in the lower section.

Assuming that these flow targets will not be achieved, or using the river as the natural water conveyor will fail, this will lead to considerable smaller flows in the river and higher salt concentrations. This again will impact the potential of the river in terms of economic and tourism development. Continued use of engineered water conveyance systems such as the current East Ghor or a future West Ghor Canal will also lead to higher operation and maintenance costs. But maybe most importantly, continued flow reduction and higher salt concentrations will lead to serious limitation in terms of restoration of aquatic ecosystems and related eco-tourism, including continued loss of floristic and faunal biodiversity and the loss of opportunity to achieve a fair to high ecosystem integrity and health.

Open Access This chapter is distributed under the terms of the Creative Commons Attribution 4.0 International License (http://creativecommons.org/licenses/by/4.0/), which permits use, duplication, adaptation, distribution and reproduction in any medium or format, as long as you give appropriate credit to the original author(s) and the source, a link is provided to the Creative Commons license and any changes made are indicated.

The images or other third party material in this chapter are included in the work's Creative Commons license, unless indicated otherwise in the credit line; if such material is not included in the work's Creative Commons license and the respective action is not permitted by statutory regulation, users will need to obtain permission from the license holder to duplicate, adapt or reproduce the material. 